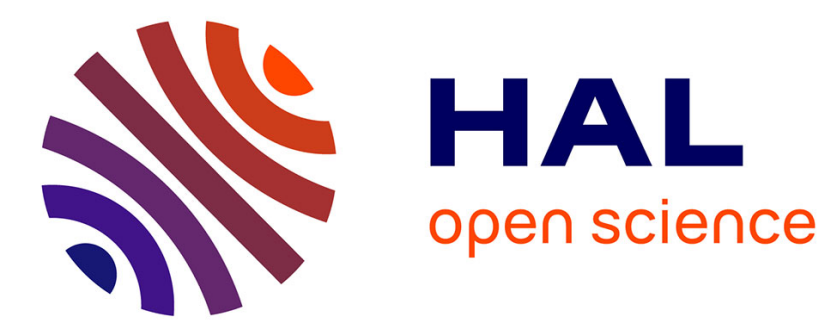

\title{
On Sato-Tate distributions, extremal traces, and real multiplication in genus 2
}

\author{
David Kohel, Yih-Dar Shieh
}

\section{To cite this version:}

David Kohel, Yih-Dar Shieh. On Sato-Tate distributions, extremal traces, and real multiplication in genus 2. Arithmetic, Geometry, Cryptography and Coding Theory, AMS Contemporary Mathematics, 2021, 2021. hal-03089551

\section{HAL Id: hal-03089551 \\ https://hal.science/hal-03089551}

Submitted on 28 Dec 2020

HAL is a multi-disciplinary open access archive for the deposit and dissemination of scientific research documents, whether they are published or not. The documents may come from teaching and research institutions in France or abroad, or from public or private research centers.
L'archive ouverte pluridisciplinaire HAL, est destinée au dépôt et à la diffusion de documents scientifiques de niveau recherche, publiés ou non, émanant des établissements d'enseignement et de recherche français ou étrangers, des laboratoires publics ou privés. 


\title{
On Sato-Tate distributions, extremal traces, and real multiplication in genus 2
}

\author{
David Kohel and Yih-Dar Shieh
}

\begin{abstract}
The vertical Sato-Tate conjectures gives expected trace distributions for for families of curves. We develop exact expression for the distribution associated to degree- 4 representations of $\mathrm{USp}(4), \mathrm{SU}(2) \times \mathrm{SU}(2)$ and $\mathrm{SU}(2)$ in the neighborhood of the extremities of the Weil bound. As a consequence we derive qualitative distinctions between the extremal traces arising from generic genus-2 curves and genus- 2 curves with real or quaternionic multiplication. In particular we show, in a specific sense, to what extent curves with real multiplication dominate the contribution to extremal traces.
\end{abstract}

\section{Introduction}

Let $C / \mathbb{F}_{q}$ be a curve of genus $g$ over the field $\mathbb{F}_{q}$ of $q$ elements and let $J$ be its Jacobian. The characteristic polynomial of the Frobenius endomorphism $\pi \in \operatorname{End}(J)$, is of the form

$$
\chi_{C}(x)=x^{2 g}-a_{1} x^{2 g-1}+a_{2} x^{2 g-2}+\cdots+a_{2} q^{g-2} x^{2}-a_{1} q^{g-1} x+q^{g} .
$$

We define the normalized Weil polynomial to be the characteristic polynomial of $\pi \otimes \sqrt{q}^{-1}$ in $\operatorname{End}(J) \otimes_{\mathbb{Z}} \mathbb{R}:$

$$
\tilde{\chi}_{C}(x)=x^{2 g}-\tilde{a}_{1} x^{2 g-1}+\tilde{a}_{2} x^{2 g-2}+\cdots+\tilde{a}_{2} x^{2}-\tilde{a}_{1} x+1,
$$

with normalized roots $\tilde{\alpha}_{1}, \ldots, \tilde{\alpha}_{g}, \tilde{\alpha}_{g+1}, \ldots, \tilde{\alpha}_{2 g}$ satisfying $\tilde{\alpha}_{j} \tilde{\alpha}_{j+g}=1$ where $\tilde{\alpha}_{j}=e^{i \theta_{j}}$. We denote the real numbers $\tilde{\alpha}_{j}+\tilde{\alpha}_{j+g}$ by $t_{j}$, and define $\left(s_{1}, \ldots, s_{g}\right)$ to be the symmetric polynomials in $\left(t_{1}, \ldots, t_{g}\right)$. We call the polynomial

$$
\prod_{i=1}^{g}\left(x-t_{i}\right)=x^{g}-s_{1} x^{g-1}+s_{2} x^{g-2}+\cdots+(-1)^{g} s_{g}
$$

the normalized real Weil polynomial of $C / \mathbb{F}_{q}$, noting in particular that $s_{1}=\tilde{a}_{1}$ and $s_{2}=\tilde{a}_{2}-g$.

The Sato-Tate conjecture for an non-CM elliptic curve $C / \mathbb{Q}$ concerns the equidistribution with respect to a Haar measure induced by the group $\mathrm{SU}(2)$ of the Frobenius angles $\theta_{1}$ or normalized Frobenius traces $t_{1}$ as $q$ varies over the reductions of $C$ over finite fields $\mathbb{F}_{q}$, and has been generalized to curves of higher genus or abelian varieties of higher dimension. In higher degree, this is expressed as an equidistribution of the tuples of Frobenius angles $\left(\theta_{1}, \ldots, \theta_{g}\right)$, of real traces $\left(t_{1}, \ldots, t_{g}\right)$ or of the symmetric sums $\left(s_{1}, \ldots, s_{g}\right)$ with respect to a induced Haar measure of an associated compact Lie group, the Sato-Tate group.

These generalizations are typically grouped together as horizontal Sato-Tate conjectures. Katz introduced a vertical Sato-Tate conjecture concerning the variation of a family $\mathcal{C} / S$ of curves, abelian varieties or exponential sums over a base scheme $S$. Specifically, the vertical Sato-Tate conjectures concerns the limit of the sets of Frobenius traces associated to the fibers point in $S\left(\mathbb{F}_{q}\right)$, as $q$ goes to infinity. One can view the horizontal Sato-Tate conjecture as a statement that Frobenius distribution for a fiber over a point $S$ follows the equidistribution of the vertical Sato-Tate distribution. Whereas the horizontal Sato-Tate conjectures are, in many instances, still open, the vertical Sato-Tate conjectures are more amenable to proof. In this work we assume the 
expected vertical Sato-Tate conjecture and as a consequence derive a qualitative distinction between extremal traces for quaternionic multiplication (QM), real multiplication (RM) and generic families, and when refering to the Sato-Tate group over a finite field we are implicitly considering the vertical aspect of these conjectures.

In what follows we consider the case of genus- 2 curves, for which the generic Sato-Tate group is USp(4). When the Jacobian is split or admits RM over the base field, the Frobenius endomorphism commutes with this structure, and the Sato-Tate group is restricted to $\mathrm{SU}(2) \times \mathrm{SU}(2)$. Such families of curves arise when the base is a cover of a Humbert surface $\mathcal{H}_{D}$ over which the RM ring is defined. The Humbert surface is a moduli space, contained in the 3 -fold $\mathcal{M}_{2}$ of moduli for genus-2 curves, classifying isomorphism classes of curves whose Jacobians admit endomorphisms by a real quadratic order of discriminant $D$. A special case is when $D$ is a square, corresponding to orders in the real quadratic ring $\mathbb{Z}[x] /\left(x^{2}-x\right) \cong \mathbb{Z} \times \mathbb{Z}$ of discriminant 1 . The embedding of the Sato-Tate group $\mathrm{SU}(2) \times \mathrm{SU}(2)$ in $\mathrm{USp}(4)$ depends on the choice of Humbert surface (in particular on its discriminant), but the induced Haar measure on Frobenius traces remains invariant of this embedding. Over a finite field, every curve $C / \mathbb{F}_{q}$ lies in the image of some Humbert surface, and the objective of this work is to understand how this stratification of the space $\mathcal{M}_{2}$ by Humbert surfaces $\mathcal{H}_{D}$ lets us understand the distributions of normalized Frobenius traces $s_{1}$ near the extremities of the Weil interval $[-2 g, 2 g]=[-4,4]$. To complete the picture of this stratification, we consider the Frobenius distributions of families with quaternionic multiplication (QM). These arise as families over Shimura curves $X^{N}$, admitting QM by an indefinite order in a quaternion algebra of discriminant $N$. Such curves appear as components of the intersections of Humbert surfaces, and give rise to the Sato-Tate group SU(2) embedded diagonally (up to conjugation) as a subgroup $\Delta \subset \mathrm{SU}(2) \times \mathrm{SU}(2)$ in a degree-4 representation.

The study of extremal traces (in particular curves with many points) has a long history, motivated by applications to coding theory and the rich mathematical structure going into their study. In the next section we introduce the notation for this study before turning to the Weyl integration formulas in Section 3, which gives an explicit form for the Haar measures induced by the symplectic groups $\mathrm{SU}(2)$ and $\mathrm{USp}(4)$. In the following Section 4, we develop precise Taylor series expressions for the Haar measure induced on the trace function $s_{1}$, and devote Section 5 to certain global expressions for the continuous distribution functions for $\mathrm{USp}(4)$ and $\mathrm{SU}(2) \times \mathrm{SU}(2)$ on $s_{1}$ obtained by Gilles Lachaud. As an application to the explicit Taylor series expansions, in Section 6, we give a qualitative comparison of the expected contribution of a generic family over $\mathcal{M}_{2}$, of an RM family over some Humbert surface $\mathcal{H}_{D}$, and of a QM family over a Shimura curve $X^{N}$. In particular we show that a Shimura curve $X^{N}$ and associated Sato-Tate group $\Delta$ gives the greatest density of extremal traces of these groups, but that a Humbert surface of small discriminant dominates the contribution to extremal traces of genus- 2 curves.

Acknowledgements. This work, presented in a previous instance of AGCT in 2015, grew out of discussions in the course of the doctoral work of the second author, jointly supervised by Gilles Lachaud and the first author. Certain results of Gilles in the direction of this work are presented in Section 5. Gilles' departure was both a deep personal and mathematical loss, and his impact continues to be felt in work such the present article and through the international AGCT workshops.

\section{Background and notation}

We consider Galois representations in $\mathrm{USp}(4)$ and its subgroups $\mathrm{SU}(2) \times \mathrm{SU}(2)$, and $\mathrm{SU}(2)$ arising from families of curves of genus 2. We assume a generic normalized Weil polynomial takes the form

$$
\widetilde{\chi}(x)=x^{4}-s_{1} x^{3}+\left(s_{2}+2\right) x^{2}-s_{1} x+1,
$$

in particular, $s_{1}$ represents the normalized trace of a general element. Under a splitting of the generic normalized Weil polynomial as

$$
\left(x-e^{i \theta_{1}}\right)\left(x-e^{-i \theta_{1}}\right)\left(x-e^{i \theta_{2}}\right)\left(x-e^{-i \theta_{2}}\right)=\left(x^{2}-t_{1} x+1\right)\left(x^{2}-t_{2} x+1\right),
$$


we consider the transformation of Haar measures induced by the changes of variables between local parameters $\left(\theta_{1}, \theta_{2}\right),\left(t_{1}, t_{2}\right)$, and $\left(s_{1}, s_{2}\right)$ on the respective domains of support $\Theta_{2}, I_{2}$ and $\Sigma_{2}$. In order to focus on the role of the discriminant of the normalized real polynomial:

$$
\left(x-2 \cos \left(\theta_{1}\right)\right)\left(x-2 \cos \left(\theta_{2}\right)\right)=\left(x-t_{1}\right)\left(x-t_{2}\right)=x^{2}-s_{1} x+s_{2},
$$

we consider $D_{0}=\left(t_{1}-t_{2}\right)^{2}=s_{1}^{2}-4 s_{2}$, and its square root $\delta_{0}=t_{2}-t_{1}$. Similarly, for a root $\pi$ of $\chi(x)$, with conjugate $\bar{\pi}=\pi^{-1}$, the relative ring extension $\mathbb{Z}[\pi, \bar{\pi}] / \mathbb{Z}[\pi+\bar{\pi}]$ has norm discriminant

$$
D_{1}=\left(4-t_{1}^{2}\right)\left(4-t_{2}^{2}\right)=\left(4+s_{2}\right)^{2}-4 s_{1}^{2}=\left(4-2 s_{1}+s_{2}\right)\left(4+2 s_{1}+s_{2}\right) .
$$

As in the above construction, we fix the relations such as $\left(s_{1}, s_{2}\right)=\left(t_{1}+t_{2}, t_{1} t_{2}\right)$, and identify $D_{0}$ and $D_{1}$ in $\mathbb{Z}\left[t_{1}, t_{2}\right]$ and $\mathbb{Z}\left[s_{1}, s_{2}\right]$, irrespective of the ring parameters over which they are defined.

The goal of this work is to investigate the asymptotic trace distributions in the neighborhood of $s_{1}=-4$ (or by symmetry, in the neighborhood of $s_{1}=4$ ). In particular we investigate the interpretation of the Haar measure on conjugacy classes via a stratification of the moduli space $\mathcal{M}_{2}$, with generic Sato-Tate group USp(4), by the Humbert surfaces $\mathcal{H}_{D}$, with generic Sato-Tate $\mathrm{SU}(2) \times \mathrm{SU}(2)$, and the contributions from Shimura curves, whose associated Sato-Tate group is a diagonal image of $\mathrm{SU}(2)$. We recall that the embeddings of these groups determined by the particular Humbert surface or Shimura curve varies by a conjugation, but the traces and integration formulas remain invariant.

\section{Weyl integration formula}

The Weyl integration formula gives an integral expression for the Haar measure on the space of Frobenius angles $\left(\theta_{1}, \ldots, \theta_{g}\right) \in \Theta_{g}=[0, \pi]^{g}$ or of the space of real traces:

$$
\left(t_{1}, \ldots, t_{g}\right)=\left(2 \cos \left(\theta_{1}\right), \ldots, 2 \cos \left(\theta_{g}\right)\right) \in I_{g}=[-2,2]^{g} .
$$

In particular we focus on $g=2$ and the distributions induced by subgroups of $\operatorname{USp}(4)$. We recall the form of the Weyl integration formula for the unitary symplectic group from Weyl [6, Theorem 7.8B] (see Katz and Sarnak [2, §5.0.4]).

TheOREM 1 (Weyl). The Haar measure induced by $\operatorname{USp}(2 g)$ on the angle space $\Theta_{g}$ is given by the formula

$$
\mu_{G}\left(\theta_{1}, \ldots, \theta_{g}\right)=\frac{2^{g^{2}}}{g ! \pi^{g}} \prod_{i<j}\left(\cos \left(\theta_{i}\right)-\cos \left(\theta_{j}\right)\right)^{2} \prod_{i=1}^{g} \sin ^{2}\left(\theta_{i}\right) d \theta_{1} \cdots d \theta_{g} .
$$

Degree 2. As a corollary, we specialize to the case $g=1$, where we find the classic distributions for the degree-2 representations of $\mathrm{SU}(2)=\mathrm{USp}(2)$ arising in the Sato-Tate conjecture for elliptic curves. To simplify notation we note $K=\mathrm{SU}(2)$ and use the same notation $\mu_{K}$ for the measure on the spaces $\Theta=[0, \pi]$ and $I=[-2,2]$, distiguishing the domain by the variable name.

Corollary 2. The Haar measure induced by $K=\mathrm{SU}(2)$ on the angle space $\Theta=[0, \pi]$ is given by the formula

$$
\mu_{K}(\theta)=\frac{2}{\pi} \sin ^{2}(\theta) d \theta
$$

and, in terms of the trace $t=2 \cos (\theta)$ in $I=[-2,2]$, the Haar measure takes the form

$$
\mu_{K}(t)=\frac{1}{2 \pi} \sqrt{4-t^{2}} d t
$$

We define the associated probability density functions on $\Theta$ and $I$ by

$$
f_{K}(\theta)=\frac{\mu_{K}(\theta)}{d \theta}=\frac{2}{\pi} \sin ^{2}(\theta) \text { and } f_{K}(t)=\frac{\mu_{K}(t)}{d t}=\frac{1}{2 \pi} \sqrt{4-t^{2}} .
$$

giving well-known density functions of the Sato-Tate conjectures: 

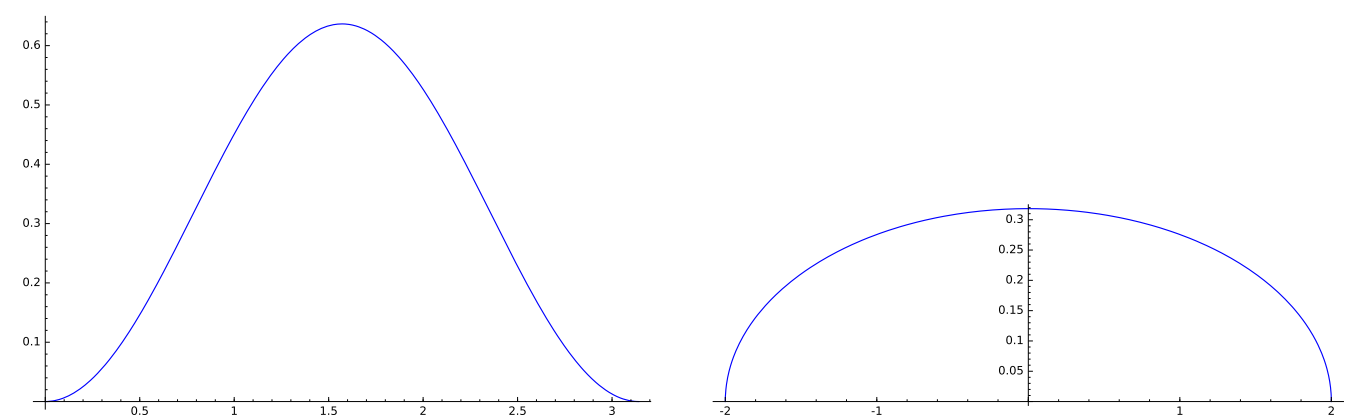

and associated cumulative density functions
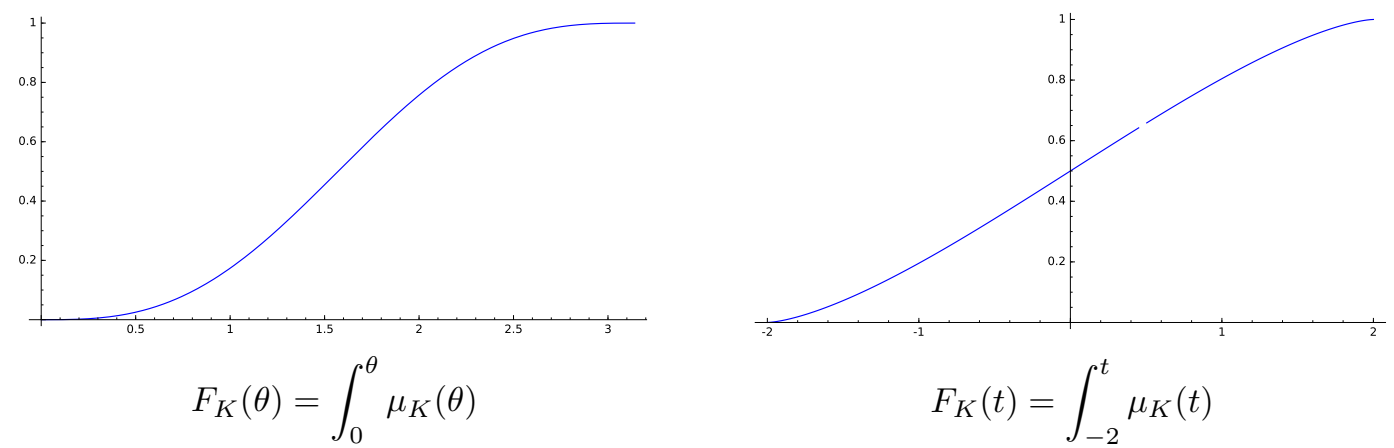

In particular, the latter distribution, on the trace space $I$, the cumulative distribution function measures the contribution of extremal traces in a neighborhood of $t=-2$, corresponding to elliptic curves with many points.

Degree 4. From the perspective of Sato-Tate distributions of genus-2 curves, we are interested in the degree- 4 representations of $\mathrm{USp}(4)$, of a subgroup $\mathrm{SU}(2) \times \mathrm{SU}(2)$, and of the image of the diagonal map $\Delta: \mathrm{SU}(2) \rightarrow \mathrm{SU}(2) \times \mathrm{SU}(2)$.

Corollary 3. The Haar measure induced by $G=\mathrm{USp}(4)$ on the angle space $\Theta_{2}$ is given by the formula

$$
\mu_{G}\left(\theta_{1}, \theta_{2}\right)=\frac{8}{\pi^{2}}\left(\cos \left(\theta_{1}\right)-\cos \left(\theta_{2}\right)\right)^{2} \sin ^{2}\left(\theta_{1}\right) \sin ^{2}\left(\theta_{2}\right) d \theta_{1} d \theta_{2} .
$$

The Haar measure for $\mathrm{SU}(2) \times \mathrm{SU}(2)$ is obtained as the product measure for the case $g=1$.

COROLlary 4. The Haar measure induced by $H=\mathrm{SU}(2) \times \mathrm{SU}(2)$ on the angle space $\Theta_{2}$ is given by the formula

$$
\mu_{H}\left(\theta_{1}, \theta_{2}\right)=\frac{4}{\pi^{2}} \sin ^{2}\left(\theta_{1}\right) \sin ^{2}\left(\theta_{2}\right) d \theta_{1} d \theta_{2} .
$$

In what follows we continue to use the notation $G$ for $\mathrm{USp}(4), H$ for $\mathrm{SU}(2) \times \mathrm{SU}(2)$, and write $\Delta$ for the diagonal image of SU(2) in $H$. Making the change of variables $t_{j}=2 \cos \theta_{j}$, such that $\sin \left(\theta_{1}\right) \sin \left(\theta_{2}\right) d \theta_{1} d \theta_{2}=d t_{1} d t_{2}$, we obtain the expression in $\left(t_{1}, t_{2}\right)$ for the Haar measure induced by $G$ and $H$ :

$$
\begin{aligned}
& \mu_{G}\left(t_{1}, t_{2}\right)=\frac{1}{8 \pi^{2}}\left(t_{1}-t_{2}\right)^{2} \sqrt{\left(4-t_{1}^{2}\right)\left(4-t_{2}^{2}\right)} d t_{1} d t_{2}=\frac{1}{8 \pi^{2}} D_{0} \sqrt{D_{1}} d t_{1} d t_{2}, \\
& \mu_{H}\left(t_{1}, t_{2}\right)=\frac{1}{4 \pi^{2}} \sqrt{\left(4-t_{1}^{2}\right)\left(4-t_{2}^{2}\right)} d t_{1} d t_{2}=\frac{1}{4 \pi^{2}} \sqrt{D_{1}} d t_{1} d t_{2} .
\end{aligned}
$$

where $D_{0}=\left(t_{1}-t_{2}\right)^{2}$ and $D_{1}=\left(4-t_{1}^{2}\right)\left(4-t_{2}^{2}\right)$. These groups of rank 2 have measure supported on the whole domain $I_{2}$. The measure for $\Delta$ is supported on the closed domain $t_{1}=t_{2}$ (or $\theta_{1}=\theta_{2}$ in $\Theta_{2}$ ). We defer the discussion of the trace distribution for $\Delta$ until after the introduction of suitable transformations of the domain. 
Domains of integration. In order to focus on the behavior of the normalized trace function $s_{1}=t_{1}+t_{2}$ of the representation in $\operatorname{USp}(4)$, and relate the measure to the coefficients of the normalized Weil polynomial, we first consider the transformation $I_{2} \rightarrow \Sigma_{2}$, where

$$
\Sigma_{2}=\left\{\left(s_{1}, s_{2}\right) \in \mathbb{R}^{2}|2| s_{1} \mid \leq s_{2}+4,4 s_{2} \leq s_{1}^{2}\right\},
$$

sending $\left(t_{1}, t_{2}\right)$ to $\left(s_{1}, s_{2}\right)=\left(t_{1}+t_{2}, t_{1} t_{2}\right)$.
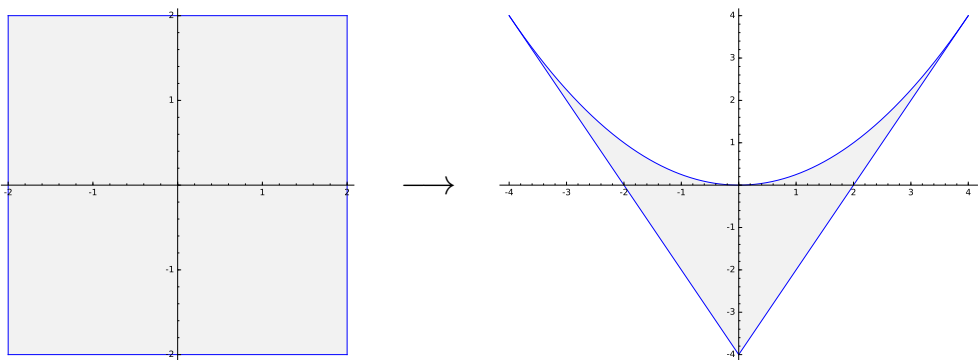

As for the discriminants $D_{i}$, we continue to use the same names for the measures $\mu_{G}$ and $\mu_{H}$ on the spaces $\Theta_{2}, I_{2}$ and $\Sigma_{2}$, distinguishing the domain by the variables names. In particular, we recall the previously determined expressions for the discriminants

$$
D_{0}=s_{1}^{2}-4 s_{2} \text { and } D_{1}=\left(4+s_{2}\right)^{2}-4 s_{1}^{2}
$$

and, in view of the equivalence of alternating volume forms,

$$
d s_{1} d s_{2}=\left(d t_{1}+d t_{2}\right)\left(t_{1} d t_{2}+t_{2} d t_{1}\right)=\left(t_{1}-t_{2}\right) d t_{1} d t_{2}=\sqrt{D_{0}} d t_{1} d t_{2},
$$

and a factor of 2 from the double cover $I_{2} \rightarrow \Sigma_{2}$, the induced Haar measure becomes

$$
\mu_{G}\left(s_{1}, s_{2}\right)=\frac{1}{4 \pi^{2}} \sqrt{D_{0} D_{1}} d s_{1} d s_{2} \text {, and } \mu_{H}\left(s_{1}, s_{2}\right)=\frac{1}{2 \pi^{2}} \sqrt{\frac{D_{1}}{D_{0}}} d s_{1} d s_{2} .
$$

In order to identify the role of the normalized discriminant $D_{0}$ of the real subring $\mathbb{Z}[\pi+\bar{\pi}]$ and with the view of obtaining a simple domain of integration for integrating over the fibers above $s_{1}$, we set $\delta_{0}^{2}=D_{0}=s_{1}^{2}-4 s_{2}$ and apply the transformation $\left(s_{1}, s_{2}\right) \mapsto\left(s_{1}, \delta_{0}\right)$ from $\Sigma_{2}$ to the domain

$$
\mathfrak{D}_{2}=\left\{\left(s_{1}, \delta_{0}\right) \in[-4,4] \times[0,4] \mid \delta_{0} \pm s_{1} \leq 4\right\} .
$$
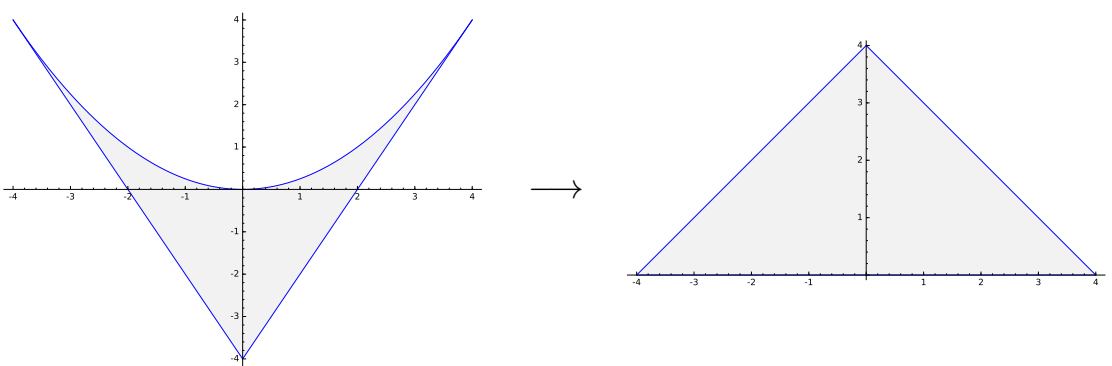

By taking the positive branch of $\delta_{0}=\sqrt{D_{0}} \geq 0$, this map is an isomorphism. Moreover, from $s_{1}^{2}-4 s_{2}=\delta_{0}^{2}$, the volume forms satisfy

$$
d s_{1} d s_{2}=\frac{1}{2} \delta_{0} d \delta_{0} d s_{1}
$$

from which we write

$$
\mu_{G}\left(s_{1}, \delta_{0}\right)=\frac{1}{8 \pi^{2}} \delta_{0}^{2} \sqrt{D_{1}} d \delta_{0} d s_{1} \text {, and } \mu_{H}\left(s_{1}, \delta_{0}\right)=\frac{1}{4 \pi^{2}} \sqrt{D_{1}} d \delta_{0} d s_{1},
$$

noting that $D_{1}$ takes the form $D_{1}=\frac{\left(\left(4+s_{1}\right)^{2}-\delta_{0}^{2}\right)\left(\left(4-s_{1}\right)^{2}-\delta_{0}^{2}\right)}{16}$.

Remark. The formal agreement of the expressions for $\mu_{G}$ on $I_{2}$ and $\mathfrak{D}_{2}$,

$$
\mu_{G}\left(t_{1}, t_{2}\right)=\frac{1}{8 \pi^{2}} D_{0} \sqrt{D_{1}} d t_{1} d t_{2} \text { and } \mu_{G}\left(s_{1}, \delta_{0}\right)=\frac{1}{8 \pi^{2}} D_{0} \sqrt{D_{1}} d \delta_{0} d s_{1} \text {, }
$$


and similarly for $\mu_{H}$,

$$
\mu_{H}\left(t_{1}, t_{2}\right)=\frac{1}{4 \pi^{2}} \sqrt{D_{1}} d t_{1} d t_{2} \text { and } \mu_{H}\left(s_{1}, \delta_{0}\right)=\frac{1}{4 \pi^{2}} \sqrt{D_{1}} d \delta_{0} d s_{1},
$$

reflects the fact that $\left(s_{1}, \delta_{0}\right)=\left(t_{1}+t_{2}, t_{1}-t_{2}\right)$ is a transformation of determinant 2 , so $d \delta_{0} d s_{1}=$ $2 d t_{1} d t_{2}$, while the domain of integration $\mathfrak{D}_{2}$ consists of points $\left(s_{1}, \delta_{0}\right)$ in the upper half of the image of points $\left(t_{1}, t_{2}\right) \in I_{2}$, such that $\delta_{0}=t_{1}-t_{2} \geq 0$.

In contrast to the above rank-2 groups, the group $\Delta$ is a rank-1 group, for which the support of the Haar measure is restricted to the 1-dimensional subspace $\delta_{0}=t_{1}-t_{2}=0$.

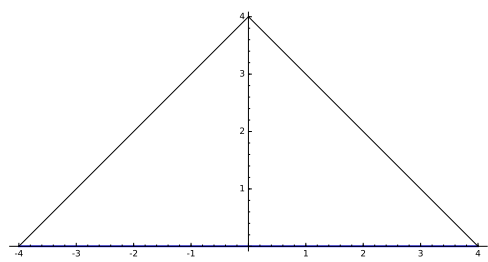

In fact, pulling back the measure for $\mathrm{SU}(2)$ by $s_{1}=t_{1}+t_{2}=2 t$ in Corollary 2 , we obtain the induced Haar measure in terms of the trace.

Corollary 5. The Haar measure induced by $\Delta$ on the trace $s_{1} \in[-4,4]$ is given by

$$
\mu_{\Delta}\left(s_{1}\right)=\frac{1}{8 \pi} \sqrt{16-s_{1}^{2}} d s_{1} .
$$

This measure can be viewed as a measure along the subdomain $[-4,4] \times\{0\} \subset \mathfrak{D}_{2}$, or as a product distribution on $\mathfrak{D}_{2}$ such that the measure in $\delta_{0}$ is a Dirac delta function with density 1 on $\delta_{0}=0$.

In what follows we seek to determine analogous expressions for the induced measure on the trace function $s_{1}$ in $[-4,4]$, coming from the rank-2 groups $G$ and $H$ by integrating in $\delta_{0}$ along the fibers about $s_{1}$. In the next section, we carry this out to develop exact series approximations for the measures in the neighborhood of $s_{1}=-4$.

\section{Taylor expansions for the trace function}

We now introduce a transformation which permits us to develop a series expansion for the Haar measure of the trace $s_{1}$ in the neighborhood of the endpoint $s_{1}=-4$. We first define the space $\Lambda_{2}=[0,4] \times[0,1]$ of points $(\varepsilon, \lambda)$ and a map $\Lambda_{2} \rightarrow \mathfrak{D}_{2}$ given by

$$
(\varepsilon, \lambda) \longmapsto(\varepsilon-4, \varepsilon \lambda) \text {. }
$$

This gives a parametrization of the left-half subspace of $\mathfrak{D}_{2}$.

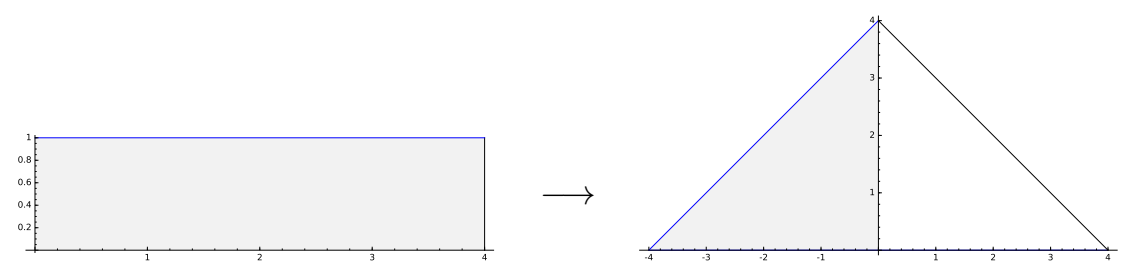

On the space $\Lambda_{2}$, we find the following expression for $D_{1}$ :

$$
D_{1}=\frac{\left(\varepsilon^{2}-\delta_{0}^{2}\right)\left((8-\varepsilon)^{2}-\delta_{0}^{2}\right)}{16}=\frac{\varepsilon^{2}\left(1-\lambda^{2}\right)\left((8-\varepsilon)^{2}-\varepsilon^{2} \lambda^{2}\right)}{16},
$$

and setting $\rho=\varepsilon /(8-\varepsilon)$ gives

$$
\sqrt{D_{1}}=\frac{\varepsilon(8-\varepsilon)}{4} \sqrt{\left(1-\lambda^{2}\right)\left(1-\rho^{2} \lambda^{2}\right)}
$$

Using $\left(s_{1}, \delta\right)=(\varepsilon-4, \varepsilon \lambda)$, it follows that $d \delta_{0} d s_{1}=(\varepsilon d \lambda+\lambda d \varepsilon) d \varepsilon=\varepsilon d \lambda d \varepsilon$, and we find

$$
\mu_{G}(\varepsilon, \lambda)=\frac{\varepsilon^{4}(8-\varepsilon)}{32 \pi^{2}}\left(\lambda^{2} \sqrt{\left(1-\lambda^{2}\right)\left(1-\rho^{2} \lambda^{2}\right)} d \lambda\right) d \varepsilon,
$$


ON SATO-TATE DISTRIBUTIONS, EXTREMAL TRACES, AND REAL MULTIPLICATION IN GENUS 2 7

and

$$
\mu_{H}(\varepsilon, \lambda)=\frac{\varepsilon^{2}(8-\varepsilon)}{16 \pi^{2}}\left(\sqrt{\left(1-\lambda^{2}\right)\left(1-\rho^{2} \lambda^{2}\right)} d \lambda\right) d \varepsilon
$$

In a neighborhood of $\varepsilon=0$, we have $\rho=\varepsilon / 8+O\left(\varepsilon^{2}\right)$, and therefore $1-\rho^{2} \lambda^{2}=1+O\left(\varepsilon^{2}\right)$, giving

$$
\mu_{G}(\varepsilon, \lambda) \approx \frac{\varepsilon^{4}(8-\varepsilon)}{32 \pi^{2}}\left(\lambda^{2} \sqrt{1-\lambda^{2}} d \lambda\right) d \varepsilon \text { and } \mu_{H}(\varepsilon, \lambda) \approx \frac{\varepsilon^{2}(8-\varepsilon)}{16 \pi^{2}}\left(\sqrt{1-\lambda^{2}} d \lambda\right) d \varepsilon .
$$

From the values of the inner integrals,

$$
\int_{0}^{1} \lambda^{2} \sqrt{1-\lambda^{2}} d \lambda=\frac{\pi}{16} \text { and } \int_{0}^{1} \sqrt{1-\lambda^{2}} d \lambda=\frac{\pi}{4},
$$

we obtain the trace distributions near $\varepsilon=0$.

THEOREM 6. The Haar measures associated to the trace function on the groups $G=\operatorname{USp}(4)$ and $H=\mathrm{SU}(2) \times \mathrm{SU}(2)$ have the following approximations at $\varepsilon=s_{1}+4=0$.

$$
\mu_{G}(\varepsilon)=\left(\frac{\varepsilon^{4}(8-\varepsilon)}{512 \pi}+O\left(\varepsilon^{6}\right)\right) d \varepsilon=\left(\frac{\varepsilon^{4}}{64 \pi}-\frac{\varepsilon^{5}}{512 \pi}+O\left(\varepsilon^{6}\right)\right) d \varepsilon
$$

and

$$
\mu_{H}(\varepsilon)=\left(\frac{\varepsilon^{2}(8-\varepsilon)}{64 \pi}+O\left(\varepsilon^{4}\right)\right) d \varepsilon=\left(\frac{\varepsilon^{2}}{8 \pi}-\frac{\varepsilon^{3}}{64 \pi}+O\left(\varepsilon^{4}\right)\right) d \varepsilon .
$$

In order to simplify compute the full Taylor expansions, we begin by recalling the definition of the Catalan numbers

$$
C_{n}=\frac{1}{n+1}\left(\begin{array}{c}
2 n \\
n
\end{array}\right)=\left(\begin{array}{c}
2 n \\
n
\end{array}\right)-\left(\begin{array}{c}
2 n \\
n+1
\end{array}\right) .
$$

noting that in particular $C_{0}=C_{1}=1$. With this definition we can state a lemma concerning the form of a class of integrals needed for our series approximation.

LEMMA 7.

$$
\int_{0}^{1} \lambda^{2 n} \sqrt{1-\lambda^{2}} d \lambda=\frac{\pi}{4} \cdot \frac{C_{n}}{4^{n}}
$$

We finally recall the form of a Taylor series for the square root.

LEMMA 8. The Taylor expansion for $\sqrt{1-x}$ is given by the power series

$$
\sqrt{1-x}=1-\frac{x}{2} \sum_{n=0}^{\infty} \frac{C_{n}}{4^{n}} x^{n}
$$

Applying Lemma 8 to $\sqrt{1-\rho^{2} \lambda^{2}}$, followed by Lemma 7 to the resulting integral summands, we obtain exact Taylor series expansions for the integrals

$$
\begin{aligned}
\int_{0}^{1} & \lambda^{2} \sqrt{\left(1-\lambda^{2}\right)\left(1-\rho^{2} \lambda^{2}\right)} d \lambda \\
& =\int_{0}^{1} \lambda^{2} \sqrt{\left(1-\lambda^{2}\right)} d \lambda-\frac{\rho^{2}}{2} \sum_{n=0}^{\infty} \frac{C_{n}}{4^{n}}\left(\int_{0}^{1} \lambda^{2(n+2)} \sqrt{1-\lambda^{2}} d \lambda\right) \rho^{2 n} \\
& =\frac{\pi}{16}\left(1-\frac{\rho^{2}}{8} \sum_{n=0}^{\infty} \frac{C_{n} C_{n+2}}{16^{n}} \rho^{2 n}\right)
\end{aligned}
$$

and

$$
\begin{aligned}
\int_{0}^{1} & \sqrt{\left(1-\lambda^{2}\right)\left(1-\rho^{2} \lambda^{2}\right)} d \lambda \\
& =\int_{0}^{1} \sqrt{\left(1-\lambda^{2}\right)} d \lambda-\frac{\rho^{2}}{2} \sum_{n=0}^{\infty} \frac{C_{n}}{4^{n}}\left(\int_{0}^{1} \lambda^{2(n+1)} \sqrt{1-\lambda^{2}} d \lambda\right) \rho^{2 n} \\
& =\frac{\pi}{4}\left(1-\frac{\rho^{2}}{8} \sum_{n=0}^{\infty} \frac{C_{n} C_{n+1}}{16^{n}} \rho^{2 n}\right) .
\end{aligned}
$$


Substituting equations (6) and (7) into equations (1) and (2) for the Haar measures arising from $G=\mathrm{USp}(4)$ and $H=\mathrm{SU}(2) \times \mathrm{SU}(2)$, gives the following theorem.

THEOREM 9. With the notation as above, the Taylor expansions for the trace on $G$ and $H$, in the neighborhood of $\varepsilon=s_{1}+4=0$ are given by

$$
\mu_{G}(\varepsilon)=\int_{\lambda=0}^{1} \mu_{G}(\varepsilon, \lambda)=\frac{\varepsilon^{4}(8-\varepsilon)}{512 \pi}\left(1-\frac{\rho^{2}}{8} \sum_{n=0}^{\infty} \frac{C_{n} C_{n+2}}{16^{n}} \rho^{2 n}\right) d \varepsilon
$$

and

$$
\mu_{H}(\varepsilon)=\int_{0}^{1} \mu_{H}(\varepsilon, \lambda)=\frac{\varepsilon^{2}(8-\varepsilon)}{64 \pi}\left(1-\frac{\rho^{2}}{8} \sum_{n=0}^{\infty} \frac{C_{n} C_{n+1}}{16^{n}} \rho^{2 n}\right) d \varepsilon
$$

Remark. As a consequence of Stirling's formula, the Catalan numbers are known to satisfy the asymptotic growth

$$
\frac{C_{n}}{4^{n}} \sim \frac{1}{\sqrt{\pi} n^{3 / 2}}
$$

from which we can conclude the convergence of the above formulas for $\varepsilon$ in the interval $[0,4]$, since $0 \leq \rho=\varepsilon /(8-\varepsilon) \leq 1$. This implies convergence for the trace $s_{1}$ in the interval $[-4,0]$, and by symmetry around $s_{1}=0$, extending to the interval $[-4,4]$.

We conclude this section with the analogous series expansion for the degree-4 diagonal subgroup $\Delta$. by

THEOREM 10. The Haar measure induced by $\Delta$ in the neighborhood of $\varepsilon=s_{1}+4=0$ is given

$$
\mu_{\Delta}(\varepsilon)=\frac{1}{\sqrt{8} \pi} \sqrt{\varepsilon(1-\varepsilon / 8)}=\frac{\varepsilon^{1 / 2}}{\sqrt{8} \pi}\left(1-\frac{\varepsilon}{16} \sum_{n=0}^{\infty} \frac{C_{n}}{4^{n}}\left(\frac{\varepsilon}{8}\right)^{n}\right) .
$$

Proof. Substituting $\varepsilon=s_{1}+4$ in Corollory 5 gives

$$
\mu_{\Delta}(\varepsilon)=\frac{1}{8 \pi} \sqrt{\varepsilon(8-\varepsilon)} d s_{1}=\frac{\varepsilon^{1 / 2}}{\sqrt{8} \pi} \sqrt{1-\varepsilon / 8} d s_{1} .
$$

Applying Lemma 8, we obtain the desired expansion.

By the previous remark, this gives convergence for $\varepsilon$ in $[0,8]$ and thus $s_{1}$ in $[-4,4]$.

We recall that the density function of the trace function on $\Delta$ is the function $f_{\Delta}(x)$ such that $\mu_{\Delta}\left(s_{1}\right)=f_{\Delta}\left(s_{1}\right) d s_{1}$. Either from the exact form of Corollary 5 ,

$$
f_{\Delta}(x)=\frac{1}{8 \pi} \sqrt{16-x^{2}}
$$

or the above series expansion, we can graph the density function $f_{\Delta}(x)$ :

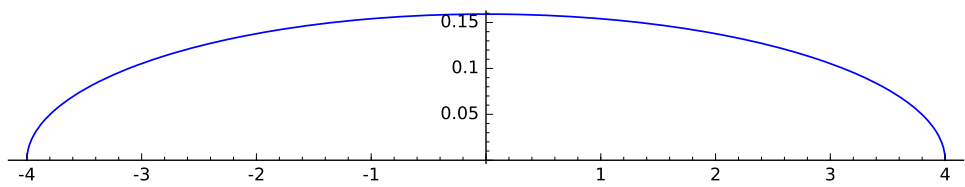

and its cumultative density function $F_{\Delta}(x)=\int_{0}^{x} f_{\Delta}(t) d t$ : 
ON SATO-TATE DISTRIBUTIONS, EXTREMAL TRACES, AND REAL MULTIPLICATION IN GENUS 29

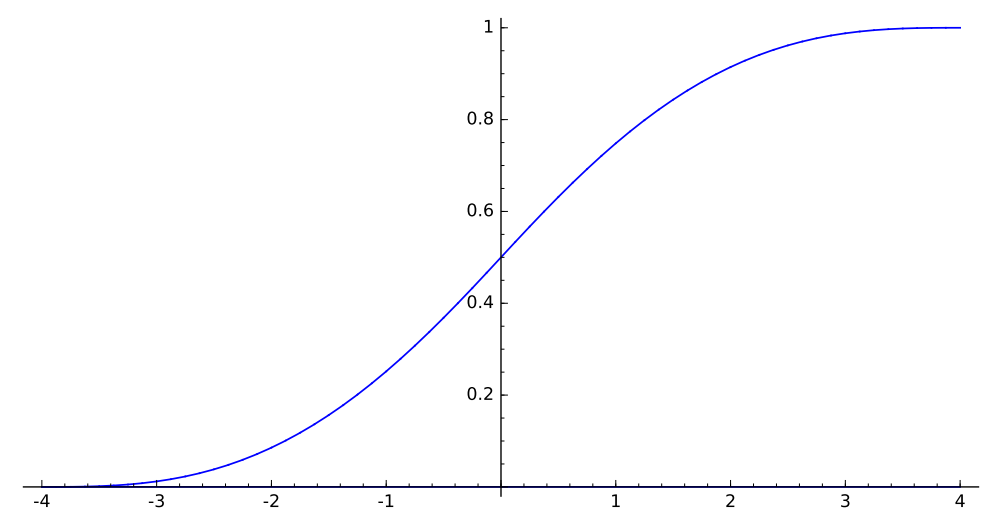

In the next section we recall results of Gilles Lachaud giving exact functional expressions for the density functions of the trace on the groups $G$ and $H$.

\section{On results of Lachaud}

In this section we detail results of Gilles Lachaud concerning the global form of the trace function for the groups $G$ and $H$, growing out of his interest in the theory of compact Lie groups and discussions with the authors during the joint supervision of the Ph.D. project of the second author. His work gives an alternative approach to develop the local expansions for the trace in the vicinity of $s_{1}= \pm 4$.

We highlight certain main results in the presentation $[\mathbf{3}]$ and preprint [4] of Lachaud. Following the notation of this work, we denote by $f_{G}(x)$ and $f_{H}(x)$ the distribution functions for the trace on $G$ and $H$ such that

$$
\mu_{G}\left(s_{1}\right)=f_{G}\left(s_{1}\right) d s_{1} \text { and } \mu_{H}\left(s_{1}\right)=f_{H}\left(s_{1}\right) d s_{1} .
$$

The first main result is an exact form for the distribution function for $G=\operatorname{USp}(4)$.

Theorem 11 (Lachaud [3]). For $G=\operatorname{USp}(4)$, if $|x|<4$, the distribution function $f_{G}$ of the trace function is given by

$$
f_{G}(x)=-\frac{64}{15 \pi} \sqrt{|x|}\left(1-\frac{x^{2}}{16}\right)^{2} \mathcal{P}_{\frac{1}{2}}^{2}\left(\frac{x^{2}+16}{4 x}\right)
$$

where

$$
\mathcal{P}_{b}^{a}(z)=\frac{\Gamma(a+b+1)}{2 \pi \Gamma(b+1)} \int_{0}^{2 \pi}\left(z+\sqrt{z^{2}-1} \cos (\varphi)\right)^{b} \cos (a \varphi) d \varphi .
$$

Remark. In particular for $z=\left(x^{2}+16\right) / 4 x$ and $(a, b)=(2,1 / 2)$, we have

$$
\mathcal{P}_{\frac{1}{2}}^{2}(z)=\frac{15}{8 \pi} \int_{0}^{2 \pi}\left(\frac{x^{2}+16+\sqrt{\left(x^{2}+16\right)^{2}-16 x^{2}} \cos (\varphi)}{4 x}\right)^{1 / 2} \cos (2 \varphi) d \varphi
$$

As a corollary, Lachaud finds an alternative expression for the trace distribution $f_{G}$ in terms of the elliptic integrals of the first kind

$$
K(m)=\int_{0}^{\pi / 2} \frac{d \varphi}{\sqrt{1-m \sin ^{2}(\varphi)}}=\int_{0}^{1} \frac{d u}{\sqrt{\left(1-u^{2}\right)\left(1-m u^{2}\right)}}=\frac{\pi}{2}{ }_{2} F_{1}\left(\frac{1}{2}, \frac{1}{2} ; 1 ; m\right)
$$

and of the second kind:

$$
E(m)=\int_{0}^{\pi / 2} \sqrt{1-m \sin ^{2}(\varphi)} d \varphi=\int_{0}^{1} \sqrt{\frac{1-m u^{2}}{1-u^{2}}} d u=\frac{\pi}{2}{ }_{2} F_{1}\left(\frac{1}{2},-\frac{1}{2} ; 1 ; m\right) .
$$

Corollary 12 (Lachaud $[\mathbf{3}]$ ). For $G=\mathrm{USp}(4)$, the distribution function $f_{G}:[-4,4] \rightarrow \mathbb{R}$ of the trace function is given by

$$
f_{G}(x)=\frac{64}{15 \pi}\left(\left(m^{2}-16 m+16\right) E(m)-8\left(m^{2}-3 m+2\right) K(m)\right),
$$


where $m=1-x^{2} / 16$.

It should be noted that the functions $K(m)$ and $E(m)$ admit well-known power series representations in $m$ :

$$
K(m)=\frac{\pi}{2} \sum_{n=0}^{\infty}\left(\frac{(2 n) !}{4^{n}(n !)^{2}}\right)^{2} m^{n} \text { and } E(m)=\frac{\pi}{2} \sum_{n=0}^{\infty}\left(\frac{(2 n) !}{4^{n}(n !)^{2}}\right)^{2} \frac{m^{n}}{1-2 n}
$$

which permits one to compute a power series representation in $m=1-x^{2} / 16$, from which one can derive series expansions around $x= \pm 4$. From the global form for $f_{G}(x)$, one can compute a graphic representation for the density function:

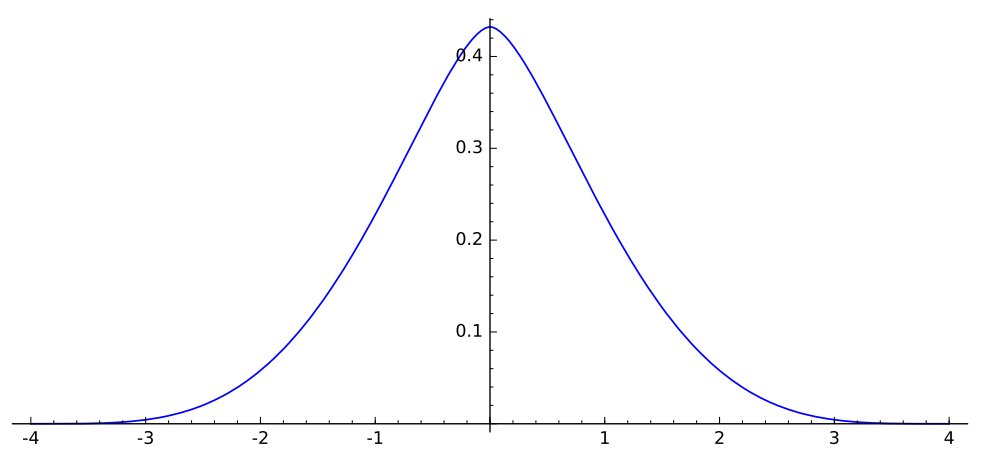

and its cumulative density function $F_{G}(x)=\int_{0}^{x} f_{G}(x) d x$ :

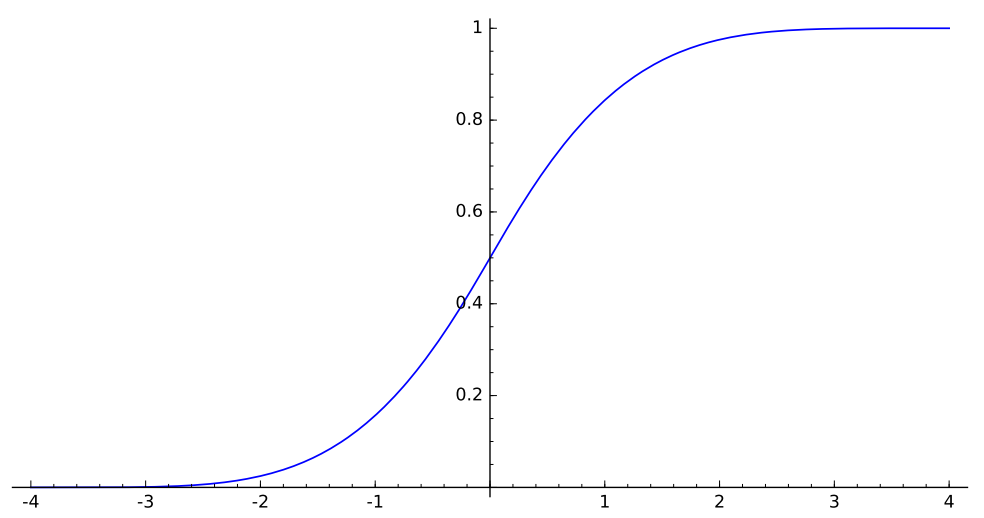

Theorem 13 (Lachaud $[4]$ ). For $H=\mathrm{SU}(2) \times \mathrm{SU}(2)$, the density function $f_{H}:[-4,4] \rightarrow \mathbb{R}$ of the trace function is given by

$$
f_{H}(x)=\frac{m^{2}}{2 \pi}{ }_{2} F_{1}\left(\frac{1}{2}, \frac{3}{2} ; 3 ; m\right),
$$

where ${ }_{2} F_{1}(a, b ; c ; z)$ is the hypergeometric function and $m=1-x^{2} / 16$.

From the explicit formula for the $f_{H}(x)$, we can compute its graphical representation:

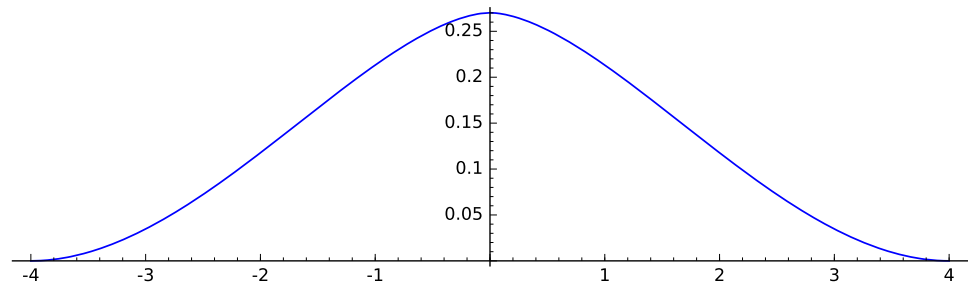

and its cumulative density function $F_{G}(x)=\int_{0}^{x} f_{H}(x) d x$ : 
ON SATO-TATE DISTRIBUtions, EXTREMAL TRACES, AND REAL MULTIPLICATION IN GENUS 2 11

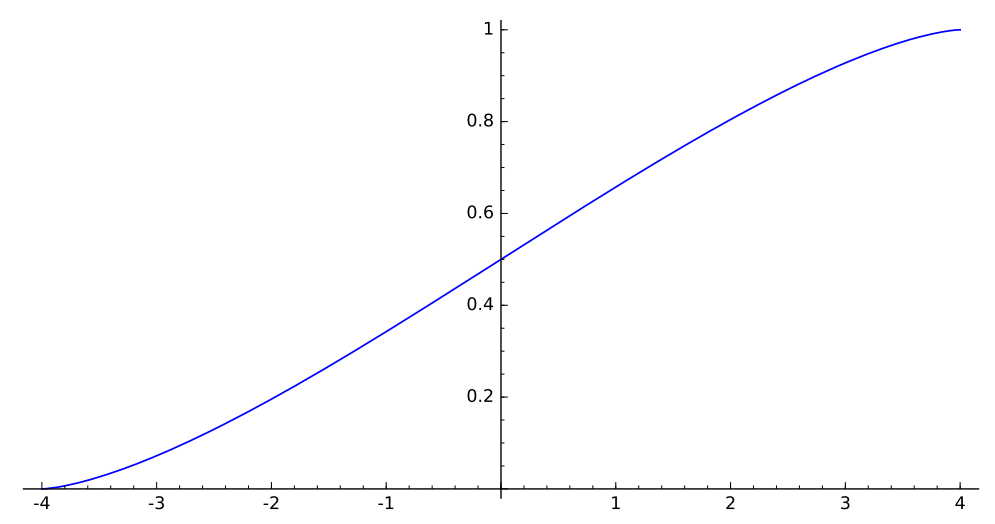

These results of Gilles Lachaud, in particular Theorems 11 and 13, give global expressions for the local power series expressions for $\mu_{G}=f_{G} d s_{1}$ and $\mu_{H}=f_{H} d s_{1}$ in Theorem 9, in terms of classical special functions. Both approaches give exact convergent expressions for the density functions $f_{G}$ and $f_{H}$ and cumulative density functions $F_{G}$ and $F_{H}$ for $s_{1}$ in the interval [-4,4], or equivalently $\varepsilon$ in $[0,8]$. In the next section we describe the application to a problem of extremal trace distributions in families which in part motived each of Gilles and the authors to carry out these respective computations of the trace distributions.

\section{Distribution of extremal traces in families}

Let $\mathcal{M}_{2}$ be the moduli space of genus- 2 curves, $\mathcal{H}_{D}$ the Humbert surface of genus- 2 curves with RM by a real quadratic order of discriminant $D$ and $\mathcal{X}^{N}$ a Shimura curve of genus-2 curves with QM by an order of discriminant $N$ in which $D$ is inert or ramified. Achter and Howe [1] determine bounds on the density of split abelian surfaces among all principally polarized abelian surfaces over a finite field of $q$ elements. Such split surfaces are accounted for by points on Humbert surfaces with $D$ a square. We are interested in the asymptotic contribution of RM and QM points among abelian surfaces, such that that the trace of Frobenius is close to the ends of the Weil interval $[-2 \sqrt{q}, 2 \sqrt{q}]$. More generally, associated to the inclusions of moduli spaces $\mathcal{X}^{N} \hookrightarrow \mathcal{H}_{D} \hookrightarrow \mathcal{M}_{2}$ we pose the question of the asymptotic contribution to extremal traces of each moduli space and the relative densities.

The respective vertical Sato-Tate groups associated with generic endomorphism ring is $G=$ $\mathrm{USp}(4)$, for real multiplication is $H=\mathrm{SU}(2) \times \mathrm{SU}(2)$ and for quaternionic multiplication is $\Delta \cong \mathrm{SU}(2) \subset H$. A family of genus- 2 curves with parametrized RM or QM endomorphism ring structure, whose base les over a Humbert surface or Shimura curve, will have trace distribution characteristic of $H$ or $\Delta$. As the respective trace distribution show (see Corollary 5, Theorems 9, 11 and 13), such families tend to have a higher density of extremal traces.

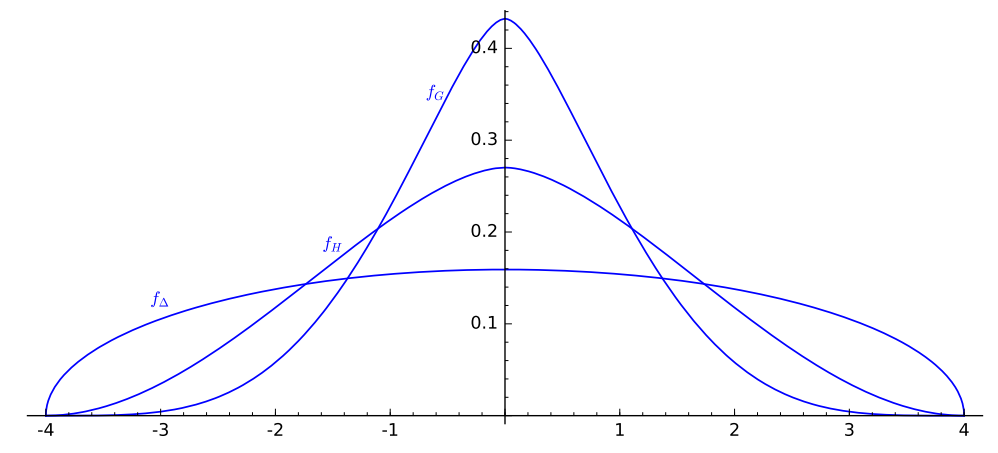

As a consequence, the cumulative density function for the trace of Frobenius in the neighborhood of $-4 \sqrt{q}$, grows faster for $\Delta$ than for $H$ and faster for $H$ than for the generic group $G$. 


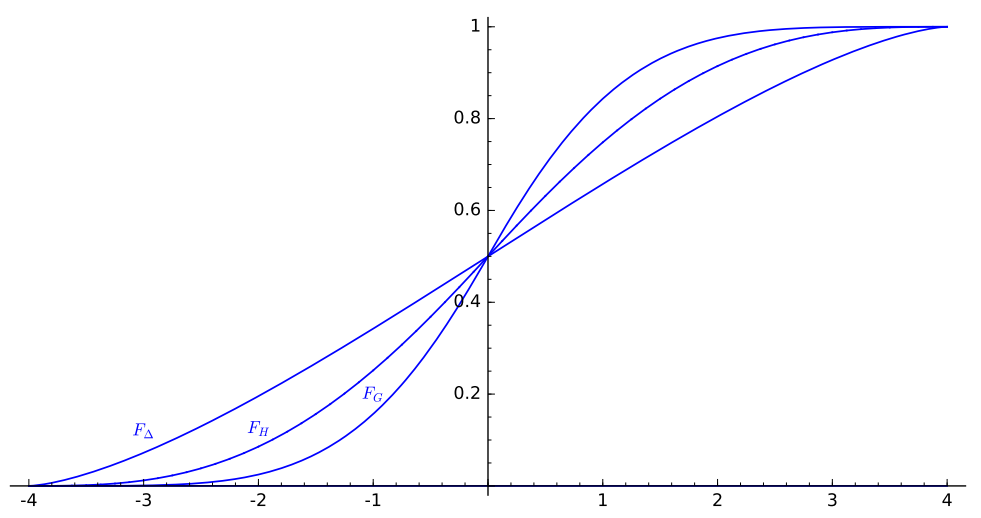

Specifically, in the following table, we summarize the first order approximations to the growth of $\varepsilon=s_{1}+4$ in neighborhood of $\varepsilon=0$ from Theorems 6 and 10, together with the associated orders of magnitude of points on the associated moduli spaces.

\begin{tabular}{|l|l|l|}
\hline$f_{G}(\varepsilon) \approx \frac{\varepsilon^{4}}{64 \pi}$ & $F_{G}(\varepsilon) \approx \frac{\varepsilon^{5}}{320 \pi}$ & $\left|\mathcal{M}_{2}\left(\mathbb{F}_{q}\right)\right| \approx q^{3}$ \\
\hline$f_{H}(\varepsilon) \approx \frac{\varepsilon^{2}}{8 \pi}$ & $F_{H}(\varepsilon) \approx \frac{\varepsilon^{3}}{24 \pi}$ & $\left|\mathcal{H}_{D}\left(\mathbb{F}_{q}\right)\right| \approx q^{2}$ \\
\hline$f_{\Delta}(\varepsilon) \approx \frac{\varepsilon^{1 / 2}}{\sqrt{8} \pi}$ & $F_{\Delta}(\varepsilon) \approx \frac{\varepsilon^{3 / 2}}{3 \sqrt{2} \pi}$ & $\left|\mathcal{X}^{N}\left(\mathbb{F}_{q}\right)\right| \approx q$ \\
\hline
\end{tabular}

Setting $\varepsilon=d / \sqrt{q}$, where $d$ is the defect from the border $t=-4 \sqrt{q}$ of the Weil interval, we observe the relative growth of the cumulative densities:

$$
\frac{F_{\Delta}(\varepsilon)}{F_{H}(\varepsilon)}=\frac{4 \sqrt{2}}{d^{3 / 2}} q^{3 / 4} \text { and } \frac{F_{H}(\varepsilon)}{F_{G}(\varepsilon)}=\frac{40}{3 d^{2}} q .
$$

Consequently a search of random points on a Shimura curve is more likely to exhibit curves of extremal trace than on a Humbert surface or for a generic curve. Every genus-2 curve with QM, however, has split Jacobian over a finite field. Similarly, a search of random points on a Humbert surface is more likely to exhibit curves of extremal trace than for a generic curve, and a general curve with moduli on a Humbert surface has absolutely commutative endomorphism ring and nonsplit Jacobian.

Considering all curves over such spaces, we find that in the neighborhood of $\varepsilon=0$, the rational points $\mathcal{H}_{D}\left(\mathbb{F}_{q}\right)$ of any single Humbert surface dominates the extremal traces within the full set of points $\mathcal{M}_{2}\left(\mathbb{F}_{q}\right)$ on the full moduli space. Specifically, with $\varepsilon=d / \sqrt{q}$, as above, we have

$$
\begin{array}{|l|}
\hline F_{G}(\varepsilon)\left|\mathcal{M}_{2}\left(\mathbb{F}_{q}\right)\right| \approx \frac{\varepsilon^{5}}{320 \pi} q^{3}=\frac{d^{5}}{320 \pi} \sqrt{q} \\
\hline F_{H}(\varepsilon)\left|\mathcal{H}_{D}\left(\mathbb{F}_{q}\right)\right| \approx \frac{\varepsilon^{3}}{24 \pi} q^{2}=\frac{d^{3}}{24 \pi} \sqrt{q} \\
\hline F_{\Delta}(\varepsilon)\left|\mathcal{X}^{N}\left(\mathbb{F}_{q}\right)\right| \approx \frac{\varepsilon^{3 / 2}}{3 \sqrt{2} \pi} q=\frac{d^{3 / 2}}{3 \sqrt{2} \pi} \sqrt[4]{q} \\
\hline
\end{array}
$$

Since $1 / 320 \pi<1 / 24 \pi$, this gives an apparent paradox for any defect $d<\sqrt{40 / 3}<3.6515$ from the end of the Weil interval. This shows clearly that the vertical Sato-Tate distribution for the trace of Frobenius on $[-4,4]$, as a limit in $q$, does not allow one to conclude existence of points in short intervals (of length $O(1 / \sqrt{q})$ ). Moreover, for particular real discriminant $D$, we prove a proposition concerning the discrete structure of moduli points in $\mathcal{H}_{D}\left(\mathbb{F}_{q}\right)$, which shows the dependence of the arithmetic of the Galois representations of Frobenius with the geometry of $\mathcal{H}_{D}$. 
Proposition 14. Suppose $A / \mathbb{F}_{q}$ is a Jacobian surface such that $\operatorname{End}(A)$ is commutative. Then there exists a unique $D$ such that the moduli point of $A$ lies in $\mathcal{H}_{D}\left(\mathbb{F}_{q}\right) \subset \mathcal{M}_{2}\left(\mathbb{F}_{q}\right)$, and the associated point $\left(s_{1}, \delta_{0}\right)$ in $\mathfrak{D}_{2}$ satisfies

$$
\delta_{0}=m_{0} \sqrt{\frac{D}{q}} \leq \varepsilon,
$$

where $\varepsilon=4-\left|s_{1}\right|$ and $m_{0}>0$ is an integer. In particular, the defect $d=\varepsilon \sqrt{q}$ with respect to the Weil bound satisfies $\sqrt{D} \leq d$.

Proof. Since $\operatorname{End}(A)$ is commutative, either $A$ is simple and $\operatorname{End}(A)$ is an order in a CM field, or $A$ is isogenous to a product of nonisogenous elliptic curves and $\operatorname{End}(A)$ is a suborder of a product of imaginary quadratic orders. Let $\operatorname{End}^{s}(A)$ be the subring fixed by the Rosatti involution, containing $\mathbb{Z}[\pi+\bar{\pi}]$. Then $\operatorname{End}^{s}(A)$ is a quadratic order of discriminant $D$. If $A$ is simple, then $\operatorname{End}^{s}(A)$ is a real quadratic order, and otherwise $\operatorname{End}(A)^{s}$ is an order the étale algebra $\mathbb{Q} \times \mathbb{Q}$ of discriminant $D=m^{2}$. The subring $\mathbb{Z}[\pi+\bar{\pi}]$ is then a suborder of index $m_{0}$ and discrimnant $m_{0}^{2} D$. In view of the optimal embedding of $\operatorname{End}^{s}(A)$ in $\operatorname{End}(A)$, the moduli point of $A$ lies in $\mathcal{H}_{D}\left(\mathbb{F}_{q}\right)$. By definition $\delta_{0}^{2} q=m_{0}^{2} D=\operatorname{disc}(\mathbb{Z}[\pi+\bar{\pi}])$, and the bounds follow since $\left(s_{1}, \delta_{0}\right)$ is a point in $\mathfrak{D}_{2}$.

Remark. The condition that $\operatorname{End}(A)$ is commutative includes all absolutely simple abelian surfaces, as well as those isogenous to a product $E_{1} \times E_{2}$ such that $E_{1}$ and $E_{2}$ are not isogenous. The latter products arise from abelian surfaces whose moduli are on Humbert surfaces of square discriminants. In contrast, any abelian surface $A$ with quaternionic multiplication by a maximal order $\mathcal{O}_{B}$ in a quaternion algebra $B$ of discriminant $N$, whose moduli point lies on a Shimura curve $X^{N}$, will have larger endomorphism ring over the algebraic closure. Over an extension such an abelian surface is isogenous to $E \times E$, where $\mathcal{O}_{K}=\operatorname{End}(E)$ is the maximal order in an imaginary quadratic field. In terms of the domain $\mathfrak{D}_{2}$, for any field $k / \mathbb{F}_{q}$ such that $\operatorname{End}_{k}(A)=\operatorname{End}(A)$, the normalized Frobenius characteristic polynomial is of the form $\left(x^{2}-t x+1\right)^{2}$, and the associated point $\left(s_{1}, \delta_{0}\right)$ satisfies $\delta_{0}=0$. Since infinitely many real quadratic orders embed in $\mathcal{O} \subset \operatorname{End}(A) \subseteq \mathbb{M}_{2}\left(\mathcal{O}_{K}\right)$, the Shimura curve lies in the intersection of infinitely many Humbert surfaces $\mathcal{H}_{D}$.

COROLlary 15. A Jacobian surface $A / \mathbb{F}_{q}$ with absolutely commutative endomorphism ring and defect $d=4 \sqrt{q}-\left|a_{1}\right|$ with respect to the Weil bound has moduli in a unique Humbert surface $\mathcal{H}_{D}\left(\mathbb{F}_{q}\right)$ with $\sqrt{D} \leq d$.

Proposition 14 asserts that the points $\left(s_{1}, \delta_{0}\right)$ in family over $\mathcal{H}_{D}$ lie in discrete bands, and consequently, there is a zone of exclusion : for $\delta_{0} \neq 0$ then

$$
\delta_{0}^{2}=s_{1}^{2}-4 s_{2}=m_{0}^{2} \frac{D}{q} \geq \frac{D}{q}
$$

Moreover, the dense set of moduli points of Jacobian surfaces with absolutely commutative endomorphism ring lie in disjoint sheets $\mathcal{H}_{D}$ (which intersect pairwise in unions of the Shimura curves of codimension 1 whose associated Frobenius representations constribute to the domain $\delta_{0}=0$ ). This gives a stratification of $\mathcal{M}_{2}$ into Humbert surfaces. The Sato-Tate distribution of USp(4) (over the generic space $\mathcal{M}_{2}$ ) can be visualized as layered in the axis $D$ by its contributions of Sato-Tate distributions for $\mathrm{SU}(2) \times \mathrm{SU}(2)$ with zone of exclusion $\delta_{0}^{2} \geq D / q$. 


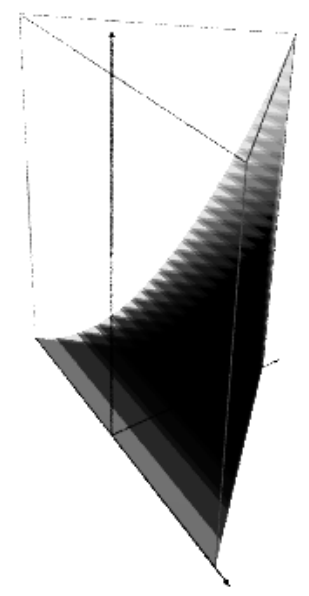

This stratification of the moduli space $\mathcal{M}_{2}$ by Humbert surfaces $\mathcal{H}_{D}$ is reflected in the precise algebraic relation between the Haar measures $\mu_{G}$ and $\mu_{H}$,

$$
\frac{\mu_{G}\left(t_{1}, t_{2}\right)}{\mu_{H}\left(t_{1}, t_{2}\right)}=\frac{\mu_{G}\left(s_{1}, s_{2}\right)}{\mu_{H}\left(s_{1}, s_{2}\right)}=\frac{\mu_{G}\left(s_{1}, \delta_{0}\right)}{\mu_{H}\left(s_{1}, \delta_{0}\right)}=\frac{D_{0}}{2},
$$

a scalar multiple of the measure $\mu_{H}$, which measures the relative number of strata $\mathcal{H}_{D}$ at a given value of $D_{0}=\delta_{0}^{2}$, contributing to the Haar measure $\mu_{G}$ over $\mathcal{M}_{2}$.

Example. The fibration of $\mathcal{M}_{2} / \mathbb{F}_{q}$ into strata $\mathcal{H}_{D} / \mathbb{F}_{q}$, and the discrete bands $\delta_{0}=m_{0} \sqrt{D / q}$ in $\mathcal{H}_{D}\left(\mathbb{F}_{q}\right)$ corresponding to each index $m_{0}=\left[\mathcal{O}_{D}: \mathbb{Z}[\pi+\bar{\pi}]\right]$ can be visualised by plotting the moduli points in $\mathcal{H}_{D}\left(\mathbb{F}_{q}\right)$. For the point $q=47$, and discriminants $D=5,12,37$ and 97 , we have:
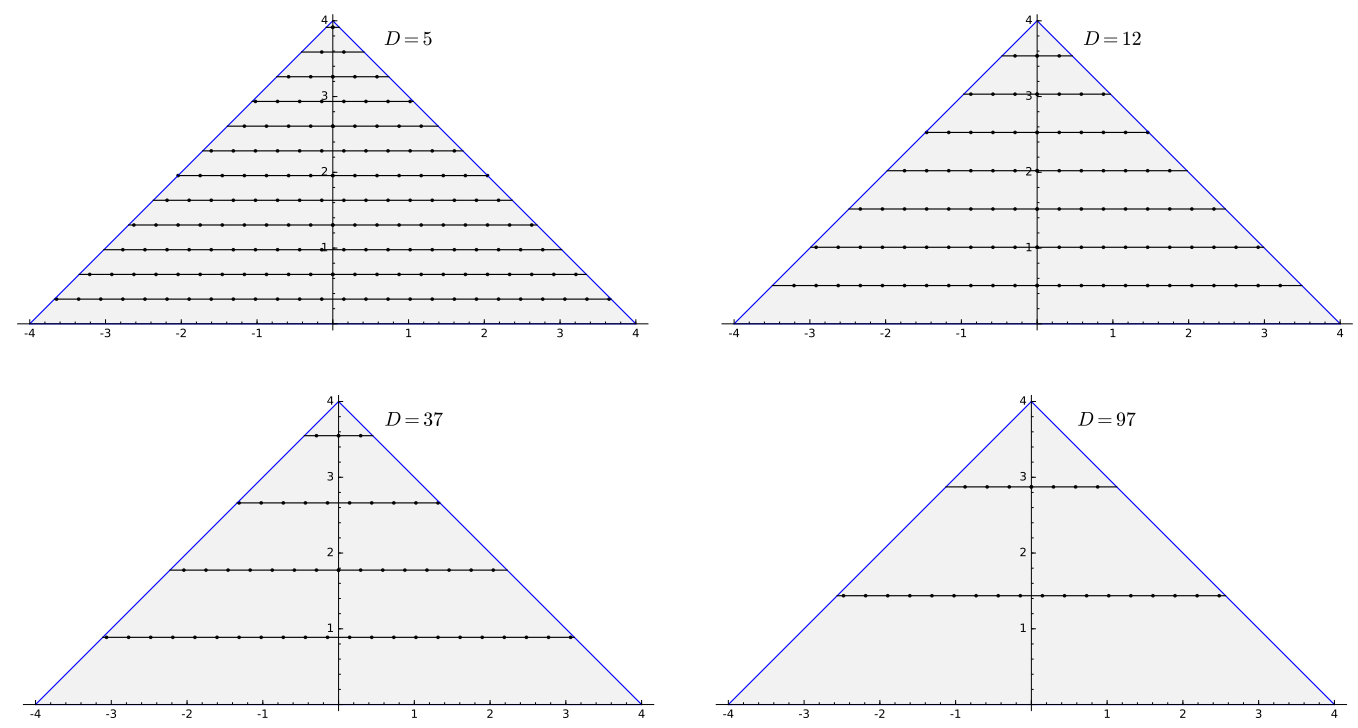

\section{Conclusion}

Based on the local expansions around the boundary of extremal traces $\left(s_{1}= \pm 4\right)$ for the degree-4 representations of $\mathrm{USp}(4), \mathrm{SU}(2) \times \mathrm{SU}(2)$ and $\mathrm{SU}(2)$, we derive explicit Taylor series expansions in the neighborhood of the extremal trace $s_{1}=-4$ (and by symmetry around $s_{1}=4$ ). In the application to curves and Jacobians over finite fields, this corresponds to genus- 2 curves with many points (and with few points, respectively). Noting that USp(4) is the generic Sato-Tate group for abelian surfaces, and $\mathrm{SU}(2) \times \mathrm{SU}(2)$ is the generic Sato-Tate group for abelian surfaces with RM, we combine this quantitative result with a qualitative analysis of the stratification of the moduli space $\mathcal{M}_{2}$ (or $\mathcal{A}_{2}$ of principally polarized abelian surfaces) by Humbert surfaces 
parametrizing RM. We use this to give a heuristic interpretation of the dominance of small RM (including split abelian surfaces) among the Jacobians of curves with many points.

An interesting question remains the role of RM and more generally exceptional endomorphisms in higher dimensions. From the Weyl integration formulas, one derives similar expressions for the induced Haar measure on $\Sigma_{g}$. As above, setting $t_{j}=2 \cos \left(\theta_{j}\right)$ and then

$$
D_{0}=\prod_{j<k}\left(t_{j}-t_{k}\right)^{2} \text { and } D_{1}=\prod_{j=1}^{g}\left(4-t_{j}^{2}\right),
$$

one finds for $G=\operatorname{USp}(2 g)$,

$$
\mu_{G}\left(s_{1}, \ldots, s_{g}\right)=\frac{1}{(2 \pi)^{g}} \sqrt{D_{0} D_{1}} d s_{1} \cdots d s_{g},
$$

and for $H=\mathrm{SU}(2)^{g}$,

$$
\mu_{H}\left(s_{1}, \ldots, s_{g}\right)=\frac{g !}{(2 \pi)^{g}} \frac{\sqrt{D_{1}}}{\sqrt{D_{0}}} d s_{1} \cdots d s_{g} .
$$

This gives an expression similar to that for $g=2$ :

$$
\frac{\mu_{G}\left(s_{1}, \ldots, s_{g}\right)}{\mu_{H}\left(s_{1}, \ldots, s_{g}\right)}=\frac{D_{0}}{g !}
$$

However, unlike in the case for $g=2$, the single parameter $D=\operatorname{disc}(\mathcal{O})$ is not sufficient to uniquely characterize an order $\mathcal{O}$ in a totally real field or etale algebra. Nevertheless, the Hilbert moduli space of a totally real order $\mathcal{O}$ maps to a $g$-dimensional subspace $\mathcal{H}_{g}(\mathcal{O})$ of the $g(g+1) / 2$ dimensional moduli space $\mathcal{A}_{g}$ of principally polarized abelian varieties of dimension $g$. In general dimension we expect the ordinary points over $\mathbb{F}_{q}$ to provide the dominant contribution to the Haar measure, and such points partition themselves into unique strata $\mathcal{H}_{g}(\mathcal{O})$. For abelian 3 -folds, this gives a stratification of the 6 -dimensional space $\mathcal{A}_{3}$ by 3 -folds $\mathcal{H}_{3}(\mathcal{O})$.

While the qualitative description is analogous, the interpretation of the ratio (10) of Haar measures is not as obvious since the discriminant $D$ doesn't characterize totally real orders of rank $g>2$. Moreover the computation of an explicit Taylor series expansion for the trace to quantify this qualitative contribution would be a much more involved calculation, whose completion would nevertheless be interesting.

\section{References}

[1] J. D. Achter and E. W. Howe. Split abelian surfaces over finite fields and reductions of genus-2 curves, Algebra Number Theory, 11, Number 1 (2017), 39-76.

[2] N. Katz and P. Sarnak. Random matrices, Frobenius eigenvalues, and monodromy, Colloquium Publications 45, American Mathematical Society, 1999.

[3] G. Lachaud. Distribution asympototique du nombre de points des courbes sur un corps fini, Séminaire ATI, 28 mars 2013.

[4] G. Lachaud. Le groupe $\mathrm{SU}(2) \times \mathrm{SU}(2)$, preprint, 2014 .

[5] F. Oort. A stratification of a moduli space of abelian varieties, in C. Faber, G. van der Geer G., and F. Oort, eds., Moduli of Abelian Varieties, Progress in Mathematics, 195, (2001), 345-416.

[6] H. Weyl. Classical groups: Their Invariants and Representations, Princeton University Press, 1961. 